\title{
Sprouting "sustainability" in chemical sciences curriculum
}

\author{
Sivasubramanian Ganarajan ${ }^{1} \cdot$ Kamakshi Ashok ${ }^{2}$
}

Received: 25 May 2018 / Accepted: 17 November 2020 / Published online: 25 November 2020

(c) Springer Nature B.V. 2020

\begin{abstract}
The purpose of this paper is to present a new viewpoint on how the curricula for higher education chemistry courses can be restructured, so that students understand the various dimensions of the sustainability concept and are equipped to work chemical sciences in a profitable, clean and societal friendly way, that is synthesis of materials (which collates CHNOPS - atomic symbols) in desired architecture and disperse them after their intended use, back into their natural reservoirs. A very novel idea of "sustainable chemical science facility", encompassing a teaching module capable of imparting strong fundamental Chemistry concepts, a virtual environment for undergoing the basic industrial training and a production unit with modular reactors for resource funding is proposed here. Often research is restricted to academic qualification, and a substantial band gap exists between the microscopic realm of academic research and the macroscopic domain of industrial chemistry, which thrives on research application. This band gap can be overcome by arming young chemists with training, tools and experience to possess complete control (synthetic and functional) on the materials they produce. The conceptual facility if turned into reality will definitely be the right step towards achieving materials with sustainable functionalities. We will have learning, training and production under one roof. Students will receive theoretical, practical and real-life experience of the production in industries, making them efficiently employable at a very young age. The idea is to bridge some critical engineering concepts with core chemistry, but with a clear distinction between chemical reactor engineering and core chemistry. This paper is a brainchild of the author and is more of a policy paper, needed to be elaborately discussed in academic and industrial forums.
\end{abstract}

Keywords Sustainable $\cdot$ Chemical science $\cdot$ Innovation · Green chemistry $\cdot$ Curriculum . Systems thinking

Kamakshi Ashok

kamakshiganarajan@gamil.com

Sivasubramanian Ganarajan

g_sivasubramanian@cb.amrita.edu

1 Department of Sciences, Amrita School of Engineering, Amrita Vishwa Vidyapeetham, Coimbatore, India

2 Sprouts Montessori House of Children, 10/56, Luz Avenue Road, Mylapore, Chennai 600004, India 


\section{Introduction}

Adaptability, discrimination ability, morality and tolerance have been perhaps the spinning wheels in bestowing the beatific birth of Homo sapiens, among the millions of lives natured equally and generously by Mother Nature. This evolutionary process of mankind is a theorem proven explicitly by the now ubiquitous concept of sustainability (http://www.un.org/sustainabledevelopment/). It is the oldest wine in newest bottle. Even though the broadest accepted definition of sustainability is "to live and let live", it has many dimensions that are over looked by mere ignorance or lack of pulsating evidence. For example, environmental education and consciousness, cutting down in carbon emissions, improving of forest resources or maintenance of a satisfying lifestyle are all an integral part of a well-structured cocoon whose life line is embedded in having a feasible and continuous thinking supported by bright, unfearful and viable action. Hence, a single common problem to humanity has different solutions-some are super imposable mirror images and some are non-super imposable mirror images. Sustainability could easily be rated as the trickiest of situations faced by the twenty-first-century humans. Every human mind should vibrate in the same wavelength so that a synchronized radiation will enlighten life uniformly across the societal spectra, ensuring the right to a decent living.

A completely sustainable lifestyle may be a dream that may not come true due to intrinsic players like economics, politics, geographics and meritus Science-controlling space, direction and speed of modern-day living. Globalization is like a double agent providing warmth/comfort on one side and cold/death on the other. Sustainability can only be harvested by sowing its seeds in the most fertile of soils called education. These multifacets of sustainability should be engraved in the curriculum, so that a society with a wishful heart to sustain can be developed (Centi 2008).

\section{Chemistry and sustainability}

Simplicity of science excels in its ability to provide selfless service to lifeful creatures. The harmonious symbiosis spreads uniformly among all creatures propagating life. Chemistry, an ambassador of this service, punctuates material comfort along the life cycle contributing to the evolution of civilizations. Human tendencies have conquered the pillars of these established social organizations, threatening life on this planet. Hence, sustaining life obligates an innovative management of available resources by all stake holders in their own home domain, accomplished through a correlated thrust towards inclusive education. The complex inter-web of 'CHNOPS' (Atomic symbols) makes all that we see with our naked eyes and under the lens. Chemical science in its modern avatar tries to decode this inter-web, understand processes involved in their making and ultimately opens up with useful ingredients which qualify as sustainable applets. Chemistry manifests itself as nano-chemistry, fashioning in the broad development of essential technologies, thus orchestrating the march of human communities to the heights of civilizations (Ozin et al. 2008; Cademartiri et al. 2009). Hence, a generation of chemists inculcated with the vision of sustainable development is an exigent requirement. This requisite can be fulfilled only by practising chemistry under the sustainability umbrella (Middlecamp and Jorgensen 2011; Mahaffy and Krief et al. 2018). 
Modern chemistry came into being with the practise of obtaining organic, inorganic or hybrid products in an industrial scale with viable economics. This continued through the nineteenth and most of the twentieth century, when suddenly a realization about the state of Mother Nature engulfed the most inspired minds of the world. This steady fast thinking transferred into international conventions, fathered by United Nations, to address these world issues. The current jeopardy of the environment is attributed to population explosion, poverty, urbanization, polarized society and at times crony capitalism. Even though chemists may not have a pertinent saying in these vicious cycles, they may well contribute silently by producing and marketing sustainable materials used in day-to-day life by the masses, thus negating the intolerance generated, on the one hand, and not compromising on the quality of lifestyle, on the other.

\section{Green and sustainable chemistry}

Over the last two decades or so a lot has been said and written about sustainability in academic and industrial research. Experts have put in a plethora of ways to deepen the concept of sustainability in chemical sciences. The foremost of these is the concept of green chemistry. Paul Anastas and John Warner put forward the twelve principles of green chemistry, which form the guidelines of green chemistry research worldwide (Anastas and Warner 1998); Roger Sheldon coined the terms atom efficiency, E-factor and environmental quotient (EQ), which indicate the environment friendliness of a chemical reaction. Remarkable progress has been made in the production of green materials, through green chemistry and green engineering. Readers are advised to expert reviews and books available in the literature for a thorough understanding of the rules governing the principles of Green Chemical Science (Ameta and Ameta 2013; Marteel-Parrish and Abraham 2014; Lancaster 2010; Zuin and Mammino 2015); life cycle assessment from cradle to grave and development of sustainability indices are very vibrant areas of research nowadays (Hellweg and i Canals 2014).

The phraseology of buzzwords like safe, clean, efficient, prevention, reduce, recycle, reuse and biodegradable has been cleverly condensed by the chemical world into green and sustainable chemistries. It is often encountered that the terms 'green chemistry' and 'sustainable chemistry' are authored synonymously in the literature. Though this usage may seem very fair at the peripheral level, delving a little, divides the two. Green chemistry is only a methodology which simplifies our efforts to attain sustainability. It is only a medium and not the solution. When a chemical process strictly adheres to safe @ raw materials, solvents and processes, less @ chemicals, solvents (no solvent) and energy (use of renewable energy) and process @ catalysis, efficient and waste prevention, then it may be termed as a green chemical process (Centi and Perathoner 2003). The products obtained by these rigorous processes could be termed green materials, even though a legalized definition is unavailable. In short, a material which paves its way into society, fulfils its master's wish and slowly fritters into its natural basin, can be safely called a sustainable material.

\subsection{Difference between green and sustainable chemistry}

Perhaps the best example to differentiate the two terms is when water is used as the reaction medium. Water qualifies all the green principles and is of course the universal solvent. Fresh water is only about $1 \%$ of the global water content and is found terrestrially as lakes, 
streams and rivers to name a few and as underground fresh water aquifers. Waste treated or otherwise from industries is disposed into terrestrial fresh water bodies, and water for use in processes is pumped underground. Potable or municipal water for all domestic purposes is drawn from aquifers and sometimes is the only lifeline to masses. Monsoon failures and drought conditions have ballooned the global water crisis at an alarming rate. Now if water is chosen as the reaction medium, instead of an organic solvent, or is an integral part of the process chemistry, the process instrumentation has to be re-designed accordingly. Once production has started, then overhauling the facility to re-accommodate the organic solvent would cause extreme hardship. Hence, given the adverse societal impact (by contributing to the water stress) of a water-driven chemical process along with judgement of industrial feasibility, the use of water at an industrial scale may not be a sustainable option, if or otherwise the industry is equipped to desalination, which takes out the economic viability from the sustainability equation. So, water is green but water-driven chemistry need not be always sustainable. They are always exceptions and the literature points to certain very rare cases where ring closing metathesis (Lipshutz and Ghorai 2009), catalytic Michael addition (Carlone and Marigo et al. 2006) have been carried out in ocean water.

\section{Practising chemistry in a sustainable way}

The swift and tight urban lives create little time for the public to respond to calls of environmental ethics. This coupled with the 2016 literacy data from UNESCO Institute of Statistics which reveal that there are about 758 million adults above 15 years who cannot read or write (McDonough and Braungart 2002) plunges the populace to behave unbecoming by ethical considerations. Hence, the idea of an ideal user is illusionary. Idealist philosophy is easy to preach, but is the most difficult to adapt and practise. So, it ultimately becomes the responsibility of the producer, in this case chemists, to produce sustainable products. An ideal sustainable product is one which extracts CHNOPS in the exact required shape, size and orientation, and dissembles CHNOPS into their natural reservoirs after the intended application- cradle to cradle (http://www.uis.unesco.org/literacy/Pages/literacy-data-relea se-2016.aspx). Also, by following the $3 \mathrm{R}$ principle of reduce, reuse and recycle, we could afloat a product for a longer duration of time, thus managing to feed the natures digestive cycle well below its saturation limit. The importance of a holistic approach towards studying chemistry redesigned with systems thinking is now recognized worldwide, and a special issue was published by the Journal of Chemical education in 2019 (Mahaffy and Ho et al. 2019). The current paper is a new approach to systems thinking with an interface of chemistry curriculum with chemical industry.

\section{Green, industrial and sustainable chemistry}

From a chemist standpoint, it requires that creating a sustainable product involves strong, structured and unambiguous understanding of the chemical principles involved and absolute control over the reaction conditions, design and process. Univocal understanding of the fundamentals can be constructed in students only with similarly adaptable structured curricula which display clarity and notional thinking. To achieve the all-important goal of communicating sustainability, a paradigm shift in thought, process and implementation of these fundamental chemical principles is necessary. Traditionally materials (organics, 
inorganics, and hybrids) were all synthesized by manipulating chemical bonds with the sole aim of harnessing the product with no regards to the path followed. Now it has become necessary to concentrate on the pathway, raw materials and solvent, these being greener the better. A conscious effort to manufacture a traditional product with its inherent application through a more acceptable route is the need of the hour. This approach can be met by looking through various dimensions and hence is prismatic (energy, reaction time, solvent, raw materials and the retail price being the different followable parameters).

\section{Alloying of the concept of sustainability into the curriculum}

Green chemistry is no new branch of chemistry, but an innovative thought process where fundamental principles are regrouped and executed, so as to satisfy the green principles. So, the basics are same but they are applied with different yardsticks and different combinations, examples being click chemistry and one-pot synthesis. Hence, the concept of green chemistry is an affirmative tool and perhaps a more organized sector which ultimately will open the way to sustainable chemical practises facilitating, better acceptable products (García-Martínez and Serrano-Torregrosa 2015). Another common perception is that green chemistry is concerned with innovative fundamental chemistry and sustainability with industrial chemistry. This also means that green chemistry is characteristic with academic research (research in educational Institutions, which grant degrees), which are done in a testing (pilot) scale in simple batch reactors. Sustainable chemistry is representative with large-scale synthesis; hence, there is a notion that such industrial processes only cause pollution and only they needed to be regulated. But as stated before, green chemistry is the pathway to attain sustainability and hence requires regulation for traversing through the microscopic domain of academic research to the macroscopic domain of industrial chemistry.

In the spectra of academic research, we have chemistry major undergraduates to postdoctoral students who do research at different levels with different reach. The outcome of such research generally culminates with a high impact factor journal publication or in some cases filing of patent. Research is mostly funded from government agencies, private entrepreneurs and industry. The young and fresh minds doing research at educational institutions are taught the basic methodologies adapted in the field. Some very bright and fortunate students start these fundamentals with state-of-the-art facilities. A project for every student is clearly envisioned and there is ample space and well-defined funding to start with. Above all the boundless knowledge and experience of teachers and professors stand tall in their carvings to reach new heights. In short, the stage is perfectly set for intellectual exuberance to win over very narrow sights of extreme sieving. This versatility and manoeuvrability of these youths make them a superb recipe for inquisitive enquiries into real-world problems. Green chemistry courses are now offered to all chemistry majors worldwide, emphasizing the need to apply these principles in their research. Thus, accepted reaction pathways, synthesis of new molecules, new chemical reactions are all contributed to the primary chemical literature from these educational institutes. Government and private owned research laboratories also contribute significantly towards the primary chemical literature adapting to green principles. Since these laboratories run only research programs and do not offer chemistry major courses, they fall outside the ambit of this paper.

The chemical industry synthesizes bulk and fine chemicals alike. The chemistry practised by these large corporates is based on successful business models where sustainability 
runs hands on with economic operability. This mantra of sustainability has revolutionized the chemical factories, an outstanding example being the $\mathrm{F}^{3}$ concept, which means fast, future and flexible chemical factories. This was based on the visionary document of European Technology Platform on Sustainable Chemistry (ETP SusChem). The $\mathrm{F}^{3}$ consortium has 25 members from both the academia and industry. It was started in 2009 with a budget of $€ 30 \mathrm{~m}$ for four-year duration. The main aim was to deliver a new 'plug and play' modular chemical production technology capable of widespread implementation throughout the European chemical industry, with enough emphasis given to process intensification and reducing the lead time from raw materials to product, thus responding quickly to market fluctuations (http://www.f3factory.com/); SPIRE (Sustainable Process Industry through Resource and Energy Efficiency) is another such example (https://www.spire2030.eu/). The vicissitudes that have occurred in chemical reactors and thereafter the functioning of chemical factories are a clean path for the academic community to follow suit. This premise refreshes the industrial domain in the vibrant practise of sustainability.

The cornerstones to the success of such industrial initiatives are the application of the process intensification techniques both equipment and methods, and catalytic technology. Both these concepts have traditionally been placed under the chemical engineering discipline. About $95 \%$ of chemical industry production is based on heterogeneous catalysis and hence is of paramount importance to the chemical fraternity. Catalysis is widely regarded as one of the extended arms of green chemistry. Even though chemistry students are taught about catalytic phenomenon and also about the chemistry behind the functioning of these systems, the technology is kindly excluded. The student knows about the electronic, geometric and structural aspects of the active catalytic molecule or species but is in dark when it comes to the shape, organizational setup inside the reactor and process related issues. Let us consider a situation in which, ideally a material qualifies to become a heterogeneous catalytic support and the active molecule is anchored upon to generate the final catalytic system. Students are accustomed to glass batch reactors to carry catalysed reaction. Even if parameters like choice of solvent, pressure and temperature are optimized, the reaction may not proceed because of high reaction volume-to-surface area ratio. Such a situation can be readily addressed if a micro-channel reactor is used because they provide very low reaction volume-to-surface area ratio. So, a reaction that does not take place in a beaker can take place in a micro reactor. Instrumentation makes the reaction happen rather than the chemistry. Also, there is a great deal of ignorance in students about the adaptability of their prepared catalyst into large-scale production. Money and precious time are getting wasted because the student's research output is not reaching any logical conclusions. Even though the research is supervised by trained faculty, students find it difficult to persuade them, though their basic chemistry is right. The supervisor tries to follow the curriculum, since at the end it will be the marks/grades that will count on the student's career in this intensely competitive world. The chemistry practised is green but is not sustainable. Therefore, a rethink in the design of chemistry curriculum globally is very necessary. The standard, method and content of teaching chemistry major will vary with different countries and universities around the world. But the syllabus taught to chemistry majors at the undergraduate or postgraduate level is more or less the same with variations only in the electives offered.

Chemistry majors are taught inorganic, organic and physical chemistries at different levels spanning the entire duration of course. At the undergraduate level, fundamentals are taught and at the postgraduate level advanced level topics with a flair to research is introduced. If these students are exposed to how chemistry is practised in industry-real hands on experience-it will improve their basic research aptitude and initiate them into 
result-oriented research. The idea here is not to integrate chemical engineering with chemistry. It will be well suited if both the disciplines are kept separate, so that there is more than equal distribution of the content, and the knowledge imparted is cohesive rather than being unconnected.

\section{The modus operandi}

\section{1 (a) A theoretical background}

The preceding paragraphs point to a noticeable gap in the way chemistry is practised by the major students and the industry, the gap being more functional (the way chemistry is practised) in nature, and can be resolved. Currently, this functional gap is bridged by regular tours or industrially exposed projects. This is currently played out by regular tours or projects taken up by students to the industry. This is a cumbersome option since more often than not student's exposure in the industry is very much limited. Hence, we need to find a mechanism where students get acclimatized to the working of chemistry in industry. This can only be achieved by the following.

1. Have two separate compulsory theory core courses: one in process intensification (equipment \& method) and another in catalyst technology. Care should be taken that the students are not over weighed in the engineering side (like design and modification) and familiarism with reactors (at a secondary level) and standard industrial practises should be the goal. These may include.

(a) Exposure to various reactors in the industry (instrumentation, advantages \& drawbacks, the chemical processes use that them and future potential applications).

(b) Current state of affairs and changes happening in the industry has to be definitely incorporated in the syllabus - at least to 3 preceding years (data from the secondary literature).

(c) Various types of catalysis, materials which qualify as supports, methods of catalysts preparation, Characterization of catalysts, mechanism of different catalytic phenomenon, role of catalyst and their reactor integration have to be taught.

(d) Selective case studies to be discussed.

(e) Wherever enzyme catalysed chemical transformation is the sole industrial method, these should also be included in the syllabus.

2. A basic chemical engineering theory course can be added as an elective.

If a theory course including the above is developed, then chemistry majors' students will definitively be in a position to restructure their green chemistry methods with a definite eye on reactor compatibility. Their research will become more time efficient, controlled and relevant to the society.

\section{2 (b) New facility for sustainable chemical science}

Theory is to be regarded as a good tested proposition only when it compliments experimental or practical results. Likewise, only lecturing students on reactors and catalysis may not give the necessary flip required to enhance their casting skill, thus eliminating 
their eligible space in the organized work force sector. Just as students undergo practical classes in a regular chemistry course, an elaborate practical session can be envisioned for this course on sustainable chemistry. The only catch here is going to be the design of the laboratory. The modular reactors will form the backbone of the laboratory, and hence, funding is a major concern. The private, public and government partnership will be a counting factor in the success of establishing the facility. The industry will also have to chip in with its inputs and requirements, also contributing to the design of the reactors. Modular reactors as in the case of $\mathrm{F}^{3}$ factories concept should be designed, rather than the obsolete models. Individual academic institutes will find it difficult to develop the concept, and hence, a consortium of these institutes can be set up and the laboratory can be implemented in common place based on geographical and economic considerations. A versatile virtual environment can be designed for the students to undergo the basic training, before entering the facility. This environment may include, simulation of-systems based on industrially successful sustainable processes, working of reactors in the facility, virtual experiments, kinetics of the simulated processes and influence of temperature, pressure and flow rates. Both batch and continuous flow processes have to be given equal importance, since bulk and fine chemical synthesis have to be carried out. Care should be taken to develop the environment under the GNU licence, since dissemination of the environment to under developed countries can be easily accomplished. But only confinement to virtual environment may not be a good strategy in the long run. At the same time, it would be the ideal launching pad for the students to get a feel of things they are due in course of time. These laboratories will only be successful if they are committed to full-fledged research programmes, which in turn will kindle the student's research aptitude.

Research and development centres of specialized industries follow few criteria mentioned above, are commercial establishments and are not opened up to undergraduate or postgraduate students. A comprehensive facility with the above attributes is non-existent, where teaching and training go hand in hand with a very strong flavour to research in an industrial terrain. The idea is to have a holistic facility with dual arm: 1) chemical plants capable of commercialization along with its accompanying logistics, trained manpower and market-oriented production capabilities; 2) a dedicated research fraternity with a composition of sustainable policy experts, inhouse instrumentation capacity builders, executive scientist and conventional teachers. Catalytic research will be one of the thrust research areas. It will also include a teaching module, with every staff member of the member institute staying connected with the facility. Students can spend up to three month and will have to clear a virtual test, written, oral and practical examinations. The project or mandatory dissertation for the under graduates and postgraduates can be replaced with the time in the facility. This course at the facility should be made compulsory for all chemistry major students of the participating member institutes. The operation side of the plant has to be commercialized for known sustainable processes so that revenue for the facility can be generated. Chemical engineering students will definitely be utilizing the facility on the engineering basis, and chemistry majors can contribute green chemistry routes in sustainable ways. Logistics, infrastructure and business model will have to be decided by the member institutes. It is of no doubt that such a facility will initially require large corpus funding, but it is the only way by which a long-lasting solution to the problem of sustainability can be reached.

In keeping with the goodwill of waste minimization at source, it would be very reasonable in acting the facility as a sustainable platform in the chemistry curriculum. The advantages of having such a facility will include the following, but not restricted to: 
Since academic and industrial research happens in one place, slowly the phrase-academic research will extinguish paving the way to only chemistry - the sustainable way.

A young, skilled work force with sound knowledge in basic and applied chemistry will be generated, even at the under or postgraduate level.

Improvements in reactor compatibility, design of new reactors, modular applications, proactive research on environmental regulations and improvement in cost reduction can be realized, through infuse of progressive thinking by students.

Socially sustainable high-end products can be produced in short duration of time.

In epidemics and pandemics like COVID-19, the response to urgent medical and material demands become the lifeline of societies. The response will depend on the rapid ability to synthesize therapeutics in extremely short duration of time. This is best achieved by accustoming synthesis in modular reactors developed by process intensification. Hence, facilities like the one proposed will serve as the best machinery during peace time and war time.

Students are treasure house of original inventive ideas; deadlocks can be overcome, since gradually the generational gap between policy makers and plant managers will vanish.

Industrially, medicinal chemist has to convert organic molecules into pharmaceutically acceptable ones, using catalytic routes and hence drug, pharmaceutical and fine chemical synthesis industries will be the big beneficiaries.

A complete revamp in the perception and practice of organic chemistry is likely, since energy efficient, low environment impact methods satisfying industrial standards (sustainable) will be developed, thus replacing the traditional synthetic routes.

The growing population of the world will be liberally benefitted as progressive chemists from growing economies will contribute to sustainable living at the country level.

The transformation of students to employable chemists and subsequently to trained and expertly skilled workforce will be velvety.

Understanding the industrial needs at budding stage in carrier, will help the students in shaping better carrier prospects.

Globalization can be controlled, by including local populace, thereby cutting down production cost, since a relatively cheap work force will be available.

Easy investment options for corporates with locally available manpower.

Attract more students to chemical sciences projecting high turnarounds in short durations.

One of the vexed problems in research has been repeatability of chemical reactions. This will be solved to a very large extent since standardization will take place in reactors under production scenario.

The sustainability facility is graphically depicted in Fig. 1.

\section{Econometrics and operational sustainability interactions in the proposed curriculum}

The advantages of the proposed facility are enumerated in the preceding paragraph. Environment, society and economics are regarded as the three pillars of sustainable development and hence an evaluation of the proposed facility in terms of these is mandated. The environmental aspect will be taken care by the principles of green chemistry, to be followed in product development. The societal evaluation is complex and is dependent on 


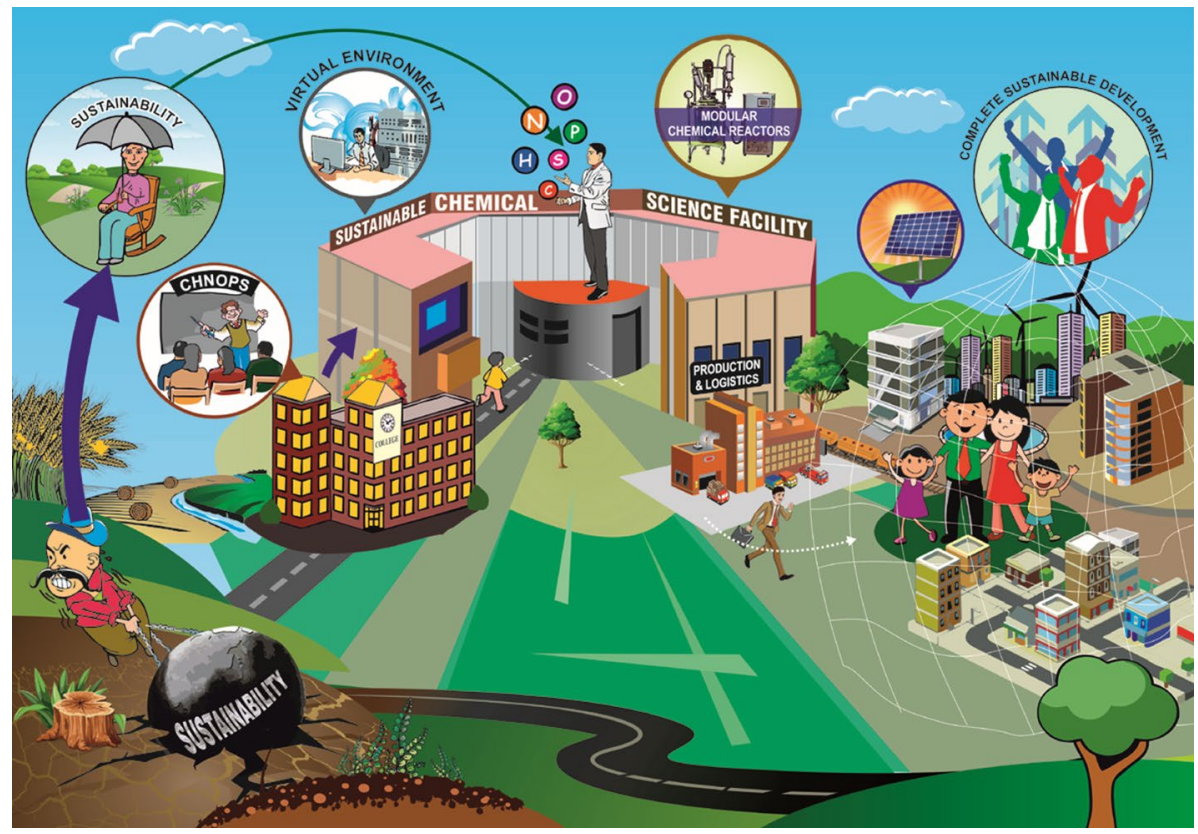

Fig. 1 Sustainability facility

many factors difficult to arrive at with quantitative descriptors, for systematic evaluation. It is assumed that the societal commitment will be fulfilled with better performance on the environment and economic fronts, since societal descriptors are difficult to frame. Since the facility is at a conceptual level, quantitative descriptors for its evaluation are intangible. Hence broader relevance of the facility to econometrics and contribution to operational sustainability, at the industrial ecology scale is discussed.

Prof. Wassily Leontief, who was awarded Nobel memorial prize in economics in 1973 did pioneering work (input-output matrix analysis) on the relation between economic growth rate and innovations (Wassily W. Leontief 1936, 1937, 1967, 1970). His work suggested that.

Rate of growth rate of GDP $=\alpha+\beta+\mathrm{Tc}$, where, $\alpha$ - Growth rate of capital used, $\beta$ Growth rate of labour or employment and Tc-Technical or Technological change.

The production curve of input-output matrix analysis is given in Fig. 2. Initially an increase in output is experienced with increase in input based on capital and labour, but further input in capital and labour does not increase the output, because of the law of diminishing returns. Hence it becomes imperative to follow different production curves with innovative ideas transforming into technologies, so that the output is not grinding to a halt.

The Nobel memorial prize in economics for 1987 was conferred upon Prof. Robert Solow. His econometric work shows that Tc/growth rate $=65 \%$ (Robert Solow 1952, 1957). This means only $35 \%$ contribution towards the overall growth rate of the GDP is due to extra capital input and additional labour and the rest $65 \%$ contribution comes from technological changes, bestowed by innovations and streamlined by market demand. He unambiguously illustrated that technological change is the driving force for increased growth rate of GDP. 
Fig. 2 Production curve with input (capital and labour) and output correlation

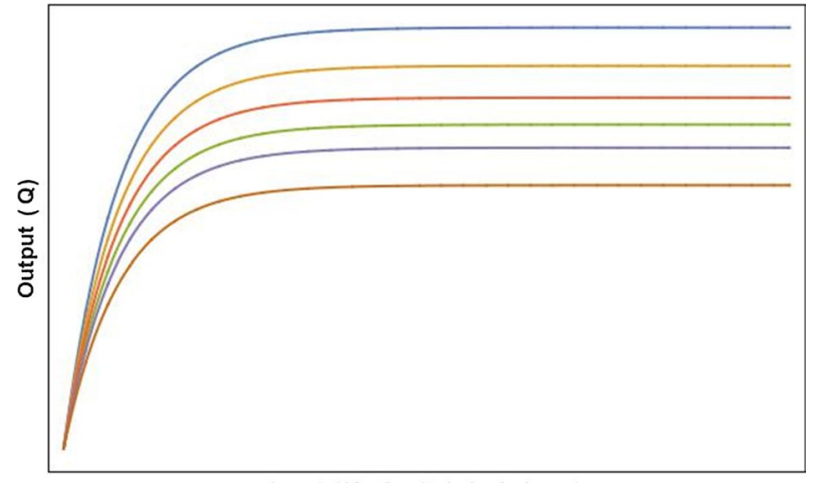

Input (K-Capital, L-Labour)

The technological change, when customized to solemnly fulfil sustainability criteria will bring about a balance in economic progress, environmental protection and societal equality.

The proposed facility will augment these findings for continuous systematic innovations, because of its capability to adhere closely to the concept of sustainability, thus developing strategic capability to adapt and rapidly respond to unprecedented circumstances and situations.

We may borrow the definition of 'Operational sustainability'- a method of evaluating whether a business can maintain existing practices without placing future potential resources at risk, or may deal with operational performance, defined as precise delivery time, better capacity utilization, and quality of supply chain,- to understand the influence of the facility in helping a manufacturing unit or a chemical science based business enterprise in taking informed decisions rapidly and adhering to green business practises. Rakesh et al. (2019) tried to correlate big data analytics with operational sustainability practises for developing better sustainable business management modules. Seven factors were involved as input to the big data analytics. They found that management and leadership style and state and central government policies are the two most important predictors of big data analytics and sustainability practices. The study confirms the hypothesis presented in this paper- introducing a curriculum at the undergraduate level, aligning perfectly with sustainable manufacture at the industrial level, put forward for the setting up of facility. By introducing the sustainability concept in the curriculum, the synthetic protocols developed by students align perfectly with those used in the industry, which means that the process taken up for industrial manufacture is already satisfying the process protocols at the industrial level and are relatively easy to implement at the policy level, which in turn provides flexibility to mid-level and lower management level players. Thus, the facility will provide a functional basis of the concept of "operational sustainability", which in turn will dissipate through the various levels of management at the industry and supply chain. In short, the management will be in position to implement stringent government legislations without changes to its raw materials procurement, plant operations, logistics and supply chain, thus easing the way to do business.

\section{Conclusions}

The idea presented in this paper is a simple scheme, which is workable. The idea of sustainability is an evolving situation that requires piercing attention at the grass root level of society. Life begins as a student and hence academia is the best place to start 
injecting this life saving scheme in the right dose and intervals. Chemistry majors are the ones who are going to shape the future of materials that the masses are going to use and hence are natural choice for the sustainability treatment. Ethical preaching in the form of lectures on pollution, environmental degradation, ideal citizenship are good beginnings, but to achieve operational sustainability, material with multi-functional facets should be manufactured through acceptable routes, so that without a great deal of changes in lifestyle, we will be able to fulfil the sustainability pledge. This will only occur when it is inherently woven in the curriculum. Student orchestration of sustainability is one of the illuminated ways for the effective deployability of the sustainability umbrella. The development of the proposed facility for practising sustainability is a systematic approach with absolute gains satisfying industry, academia and society in ideal means along with the adaptation for the marathon voyage called civilization.

\section{References}

Ameta, S. C., \& Ameta, R. (2013). Green chemistry fundamentals and applications. CRC Press: Apple Academic Press.

Anastas, P. T., \& Warner, J. C. (1998). Green chemistry: Theory and practice. Oxford: Oxford University Press.

Cademartiri, L., Ozin, G. A., \& Lehn, J.-M. (2009). Concepts of nanochemistry. NY: Wiley.

Carlone, A., Marigo, M., North, C., Landa, A., \& Jørgensen, K. A. (2006). A simple asymmetric organocatalytic approach to optically active cyclohexenones. Chemical Communications, 47, 4928-4930.

Centi, G. (2008). Excellence in innovation. Chemsuschem, 1-2, 7.

Centi, G., \& Perathoner, S. (2003). Catalysis and sustainable (green) chemistry. Catalysis Today, 77(4), 287-297.

García-Martínez J, Serrano-Torregrosa E (eds), (2015), Chemistry education: Best practices, opportunities and trends, Wiley, Verlag GmbH \& Co. KGaA.

Hellweg, S., \& i Canals, L. M. (2014). Emerging approaches, challenges and opportunities in life cycle assessment. Science, 344(6188), 1109-1113.

Lancaster, M. (2010). Green chemistry: An introductory text. UK: Royal Society of Chemistry.

Leontief, W. W. (1936). Quantitative input and output relations in the economic systems of the United States. The Review of Economics and Statistics, 18(3), 105-125.

Leontief, W. W. (1937). Interrelation of prices output, savings, and investment . The Review of Economics and Statistics, 19(3), 109-132.

Leontief, W. W. (1967). An alternative to aggregation in input-output analysis and national accounts. The Review of Economics and Statistics, 49(3), 412-419.

Leontief, W. W. (1970). Environmental repercussions and the economic structure: An input-output approach. The Review of Economics and Statistics, 52(3), 262-271.

Lipshutz, B. H., \& Ghorai, S. (2009). PQS: A new platform for micellar catalysis. RCM reactions in water with catalyst recycling. Organics Letters, 11(3), 705-708.

Mahaffy P. G., Ho F. M., Haack J. A., Brush E. J., (2019), J. Chem. Educ 96, 12, 2679-2681.

Mahaffy P. G., Krief A., Hopf H., Mehta G., Matlin S. A. (2018), Nat. Rev. Chem., 2, 0126

Marteel-Parrish, A. E., \& Abraham, M. A. (2014). Green chemistry and engineering: A pathway to sustainability, american institute of chemical engineers. Hoboken, New Jersey: Wiley .

McDonough, W., \& Braungart, M. (2002). Cradle to cradle remaking the way we make things. US: North Point Press.

Middlecamp C. H., Jorgensen A. D., (eds), (2011), Sustainability in chemistry curriculum, ACS Division of Chemical Education, ACS Symposium Series 1087.

Ozin, G. A., Arsenault, A., \& Cademartiri, L. (2008). Nanochemistry: A chemistry approach to nanomaterials. UK: Royal Society of Chemistry.

Raut, R. D., Mangla, S. K., Narwane, V. S., Gardas, B. B., Priyadarshinee, P., \& Narkhede, B. E. (2019). Linking big data analytics and operational sustainability practices for sustainable business management. Journal of Cleaner Production, 224, 10-24.

Solow, R. (1952). On the structure of linear models. Econometrica: Journal of the Econometric Society, 20(1), 29-46. 
Solow, R. (1957). Technical change and the aggregate production function. The review of Economics and Statistics, 39(3), 312-320.

Zuin, V., \& Mammino, L. (Eds.). (2015). Worldwide trends in green chemistry education. UK: Royal Society of Chemistry.

Publisher's Note Springer Nature remains neutral with regard to jurisdictional claims in published maps and institutional affiliations. 